\title{
Image Resizing for Large Displays
}

\author{
Gwanggil Jeon \\ Department of Embedded Systems Engineering, Incheon National University \\ 119 Academy-ro, Yeonsu-gu, Incheon 406-772, Korea \\ gjeon@inu.ac.kr
}

\begin{abstract}
The purpose of the image resizing is to rescale size of image to fit display device. To achieve this goal, we present a new image resizing technique. The proposed method consists of three steps, original image, (1) self-training by least squares step, (2) image upscaling step, (3) image unsharp masking step, and result image step. Three parameters $p, k$ and $h$ are computed to provide pleasant result images. Simulation results show that the proposed method outperforms benchmark methods in terms of PSNR, MSE, and SSIM metrics. In addition, visual performance comparison indicates that the proposed method is superior to the other methods.
\end{abstract}

Keywords: image resizing, image zooming, display device, unsharp masking, least squares method

\section{Introduction}

The signal scaling is an important and basic operation in image processing. The scaling can be enlarged and decreased, which is known as resizing process [1-2]. This resizing process is widely used in multimedia tools such as medical imaging and digital photography. The goal of interpolation method is to estimate the data with a continuous signal model and then restore this function on the grid proper to the wanted scaling [3].

Recently, the resizing method is applied in various fields. For instance, image fitting in the heterogeneous displays or screens can play an important role by rescaling technique [4-5]. In general, the resizing approaches are categorized into two classes: the enlarging process and the shrinking process [7]. In both processes, interpolation technique is important. There are several interpolation approaches for image resizing. One of wellknown and simplest approaches is nearest neighbor interpolation method. In addition, bilinear interpolation method, bicubic interpolation method, and Lanczos interpolation are also widely used.

Although there are several interpolation approaches, the most important role of interpolation is to restore and preserve details in the restored image. The conventional interpolation methods cannot fully preserve the details, therefore we present a new interpolation method. There have been many applications such as deinterlacing [8-11], denoising [12-13], and demosaicking [14-15] that use interpolation methods.

In this paper, we proposed a new image resizing method. Rest of the paper is organized as follows. The proposed method is presented in Section 2. In Section 3, experimental results are described. Section 4 provides conclusion remarks.

\section{Proposed Method}

The image resizing is one of key techniques to manage low resolution images to high resolution images. The flowchart of the proposed method is shown in Figure 1. The proposed method consists of five blocks and three operations. 


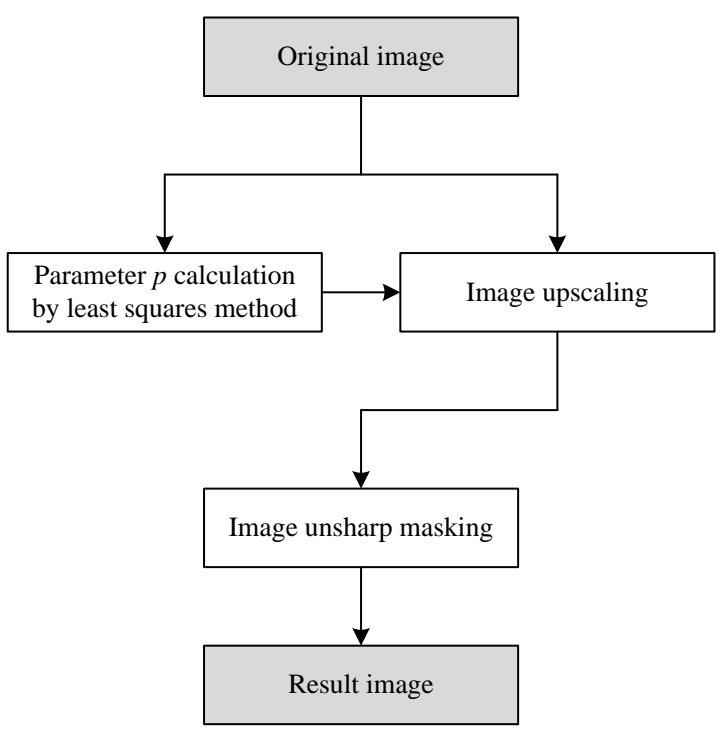

Figure 1. Flowchart of the Proposed Approach

Let us assume that the low resolution original image $\mathrm{A}_{\mathrm{i}, \mathrm{j}}$ of size height $\times$ width straightly acquired from of size of 2 height $\times 2$ width, then $\mathrm{B}_{2 \mathrm{i}, 2 \mathrm{j}}=\mathrm{A}_{\mathrm{i}, \mathrm{j}}$. Here, $\mathrm{B}_{2 \mathrm{i}, 2 \mathrm{j}}$ is result high resolution image. Now, the problem is how we can interpolate the interlacing lattice $B_{2 i+1,2 j+1}$ from the lattice $B_{2 i, 2 j}=A_{i, j}$. In other words, calculating parameter $p$ is one of goals of this work. Equation (1) shows the basic form of the image upsampling.

$$
B_{2 i+k, 2 j+l}=\sum_{k=0}^{1} \sum_{l=0}^{1} p_{2 k+l} B_{2(i+k), 2(j+l)}
$$

If we assume that the given images can be considered as locally stationary, independent and identically distributed Gaussian model, then by standard least squares methods parameter $p$ can be computed as

$$
\vec{p}=C^{-1} \vec{c}
$$

where $\mathrm{C}=\left[\mathrm{C}_{\mathrm{kl}}\right]$ and $\vec{c}=\left[\mathrm{c}_{\mathrm{k}}\right]$ are local covariances at the high resolution image. By applying Eq. (1) and Eq. (2), image upscaling is performed. This technique can be widely used such as in image deinterlacing, denoising and demosaicking, where one can magnify the size of grayscale or multichannel images.

After image upsampling is performed, we apply unsharp masking (UM). The UM process is an image enhancement method. The upsampling process may include interpolation and estimation. However, as the original image size is half of result image, this procedure is ill-posed. In addition, the upsampled image is generally blurred as edge details are losing. Thus, the UM process enhances an image which is less blurry than the upsampled image. This process is performed as,

$$
\begin{aligned}
B & =B+\{B-\operatorname{lpf}(B)\} \cdot k \\
& =(1+k) B-\operatorname{lpf}(B) \cdot k
\end{aligned}
$$

where $k$ is the amount parameter which controls the UM effect. If $k$ is bigger value, more UM effect is applied. The command lpf is low pass filter, and $\operatorname{lpf}(\mathrm{B})$ is result image after low pass filtering. We assume lpf filter is Eq. (3). Parameter $B$ is the UM effect

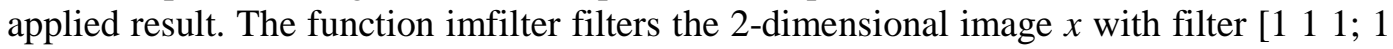
$11 ; ; 111] /(h+8)$. In this paper, parameters $k$ and $h$ are set to 0.5 and 2. Figure 2 compares original blurred image and its corresponding UM process image. 


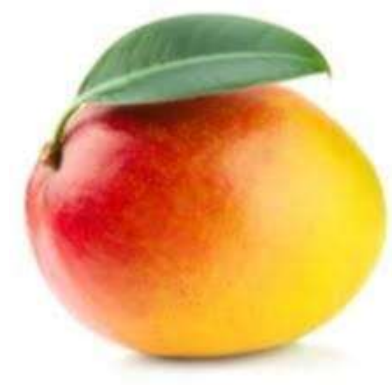

(a)

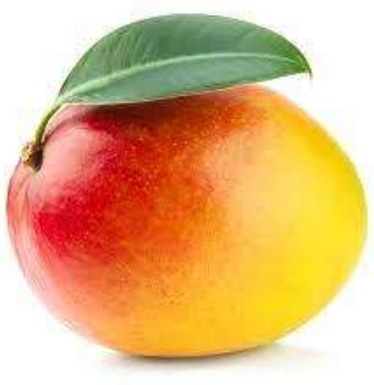

(b)

Figure 2. (a) Original 'Mango' Image. (b) UM Processed 'Mango' Image

$$
\operatorname{lpf}(x)=\text { imfilter }\left(x, \frac{1}{h+8}\left[\begin{array}{lll}
1 & 1 & 1 \\
1 & h & 1 \\
1 & 1 & 1
\end{array}\right]\right)
$$

\section{Simulation Results}

In this Section, to prove the quality of the presented approach, we tested experiments using MATLAB software with a processor of Intel(R) Core(TM) i5 CPU M460 @ 2.53GHZ. We used twenty LC dataset, between \#1 to \#20 images. Figure 3 shows twenty LC images. The size of test image is $720 \times 540$. For visual performance comparison, we used three benchmark methods, nearest neighbor (NN), bilinear (BI), and bicubic (BC) methods. We used three quality assessment tools: mean squared error (MSE), peak signal-to-noise ratio (PSNR), and structural similarity (SSIM) metrics.

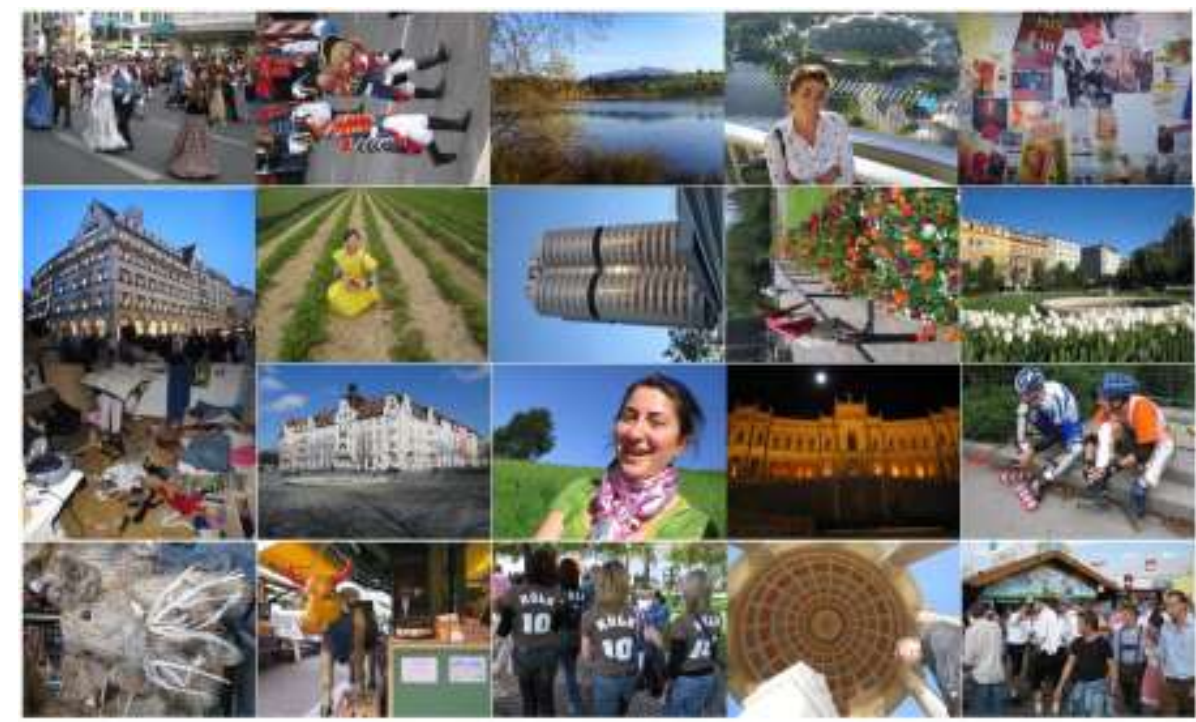

Figure 3. Test Image Sets: LC Dataset. \#1-\#20 Images are Used in the Simulation

To assess the performance of objective quality and visual performance, three metrics were used. The PSNR is computed as followed,

$$
P S N R=10 \times \log _{10}\left(\frac{M A X^{2}}{M S E}\right)
$$


where MSE denotes the mean squared error and MAX stands for the maximum possible intensity of the given image. When a pixel is represented using $n$ bits per sample, MAX is $2^{n}-1$. In general, acceptable PSNR level is between 30 to $50 \mathrm{~dB}$ for 8 bits images. For the case of 16 bits images, 60 to $80 \mathrm{~dB}$ is desirable. The MSE is mean squared error, which is computed as,

$$
M S E=\frac{1}{M N} \sum_{m=0}^{M} \sum_{n=0}^{N}[\operatorname{ori}(m, n)-\operatorname{res}(m, n)]^{2}
$$

where ori and res stand for original and the result images.

The SSIM index is invented to assess the quality between the original and the restored images. The SSIM is used to estimate the similarity between two given images. As original image is used for assessment, SSIM is a full reference metric. The SSIM is intended to enhance the conventional quality assessment tools such as PSNR, MSE.

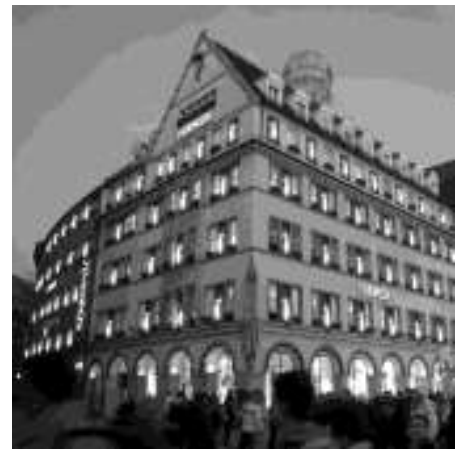

(a)

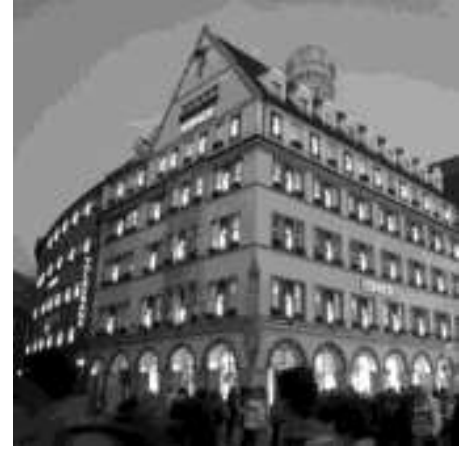

(d)

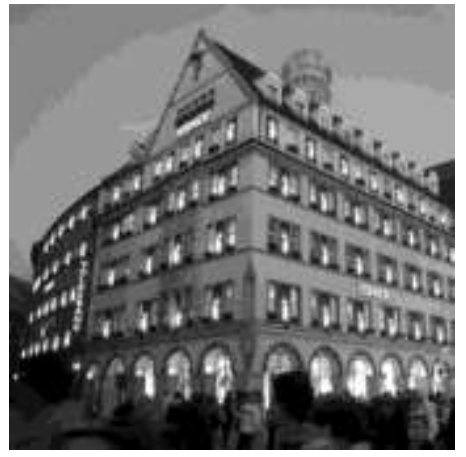

(b)

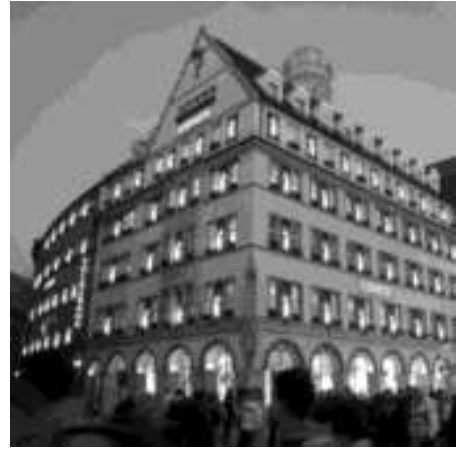

(c)

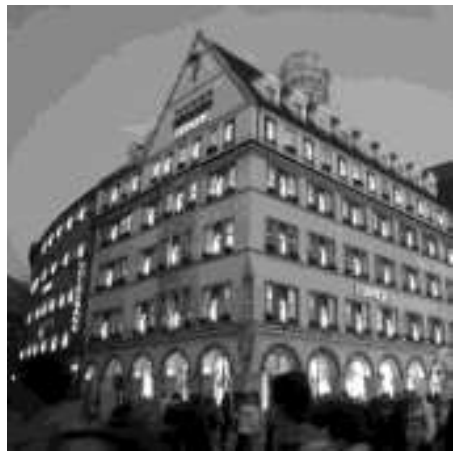

(e)

Figure 4. Visual Performance Comparison on \#6 LC Dataset. (a) Original Image, (b) Result of Nearest Neighbor Method, (c) Result of Bilinear Method, (d) Result of Bicubic Method, and (e) Result of Proposed Method

The restored images are displayed in Figs. 4-6. In all figures, images (a) indicate original cropped images with 256x256 size. In the same manner, images (b), (c), (d), and (e) are the results by nearest neighbor method, bilinear method, bicubic method, and the proposed method. All figures show that the proposed method generates subjectively pleasant results when compared to the benchmark methods. For example, the staircase artifices are shown in nearest neighbor result, blurring effect are shown in bilinear and bicubic, while the proposed method well preserved details. This can be found in window and door areas in Figure 4. 


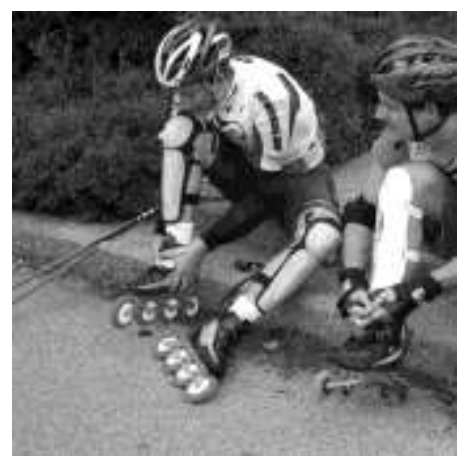

(a)

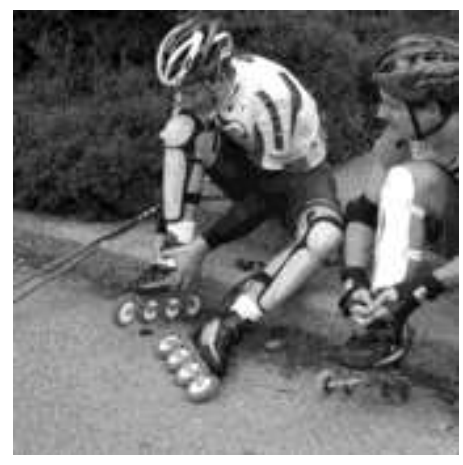

(d)

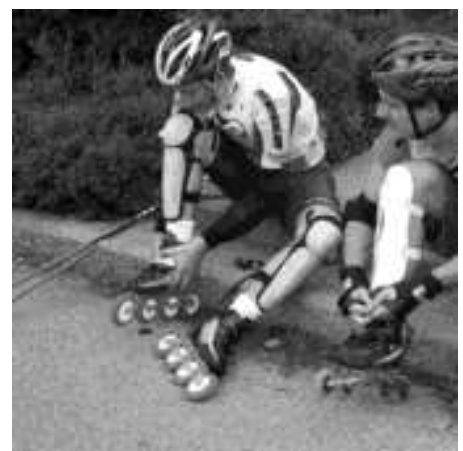

(b)

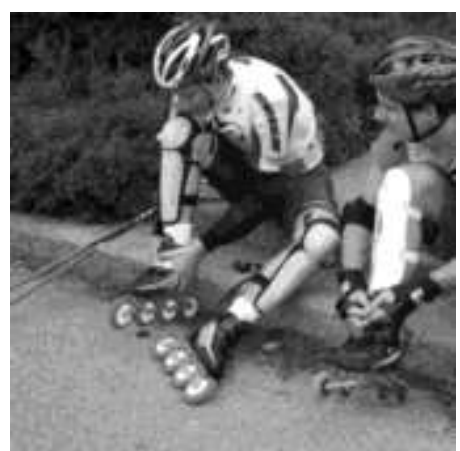

(c)

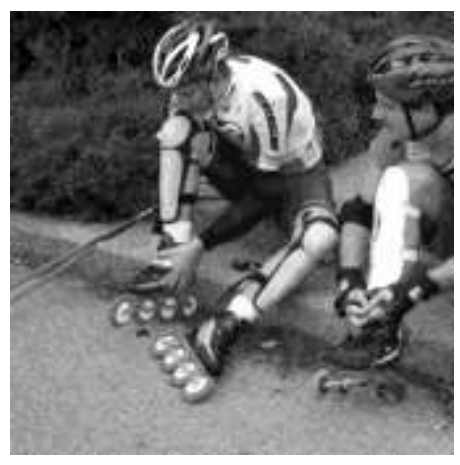

(e)

Figure 5. Visual Performance Comparison on \#15 LC Dataset. (a) Original Image, (b) Result of Nearest Neighbor Method, (c) Result of Bilinear Method, (d) Result of Bicubic Method, and (e) Result of Proposed Method

Figure 5 shows 'bikemen' image. The result image by the proposed method well restore characters on the jersey, helmet, and roller skate. Figure 6 shows roof images. Figures 6(b), 6(c), and 6(d) do not well preserve roof details, while the proposed method shows the details.

Figures 7 and 8 show SSIM results where \#1 and \#3 images were adopted for comparison. Four images (a) to (d) are results of nearest neighbor, bilinear, bicubic, and the proposed methods, respectively. In SSIM metric, the result close to ' 1 ' indicates better image while the result close to ' 0 ' indicates worse image. As one can see, the proposed method provides the best SSIM result throughout the given image. In Figure 8, SSIM value on edge area near the mountain is high. This implies that the proposed method outperforms the other methods. 


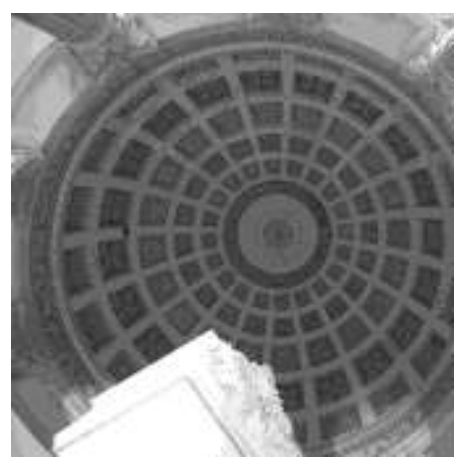

(a)

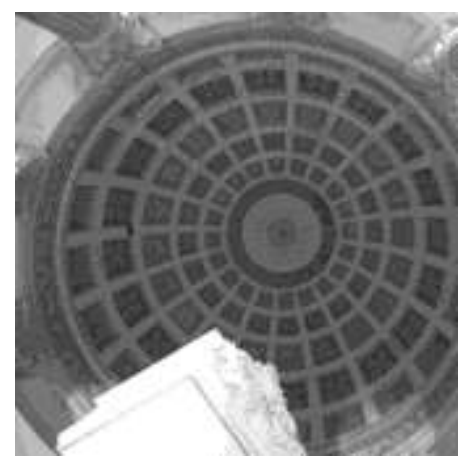

(d)

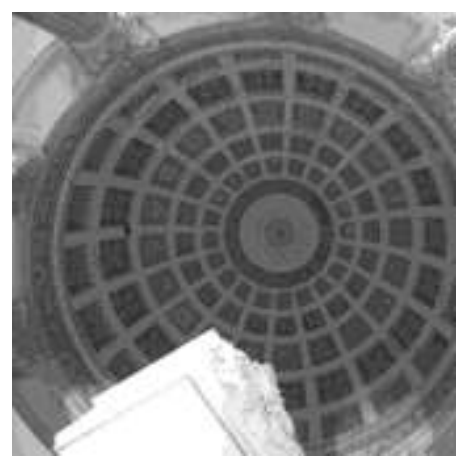

(b)

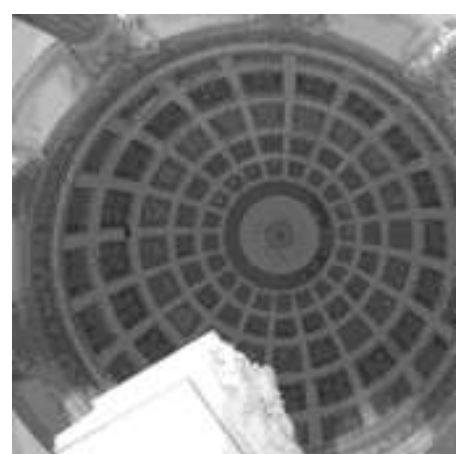

(c)

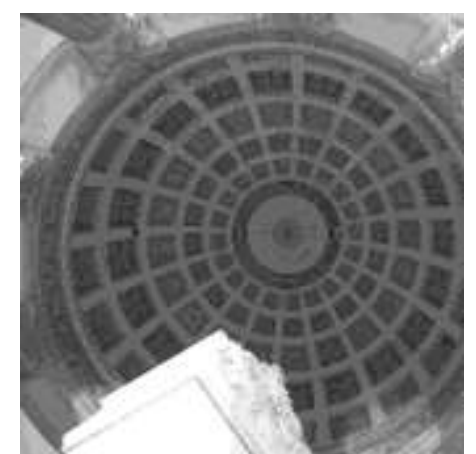

(e)

Figure 6. Visual Performance Comparison on \#19 LC Dataset. (a) Original Image, (b) Result of Nearest Neighbor Method, (c) Result of Bilinear Method, (d) Result of Bicubic Method, and (e) Result of Proposed Method

Objective performance comparisons are provided in Tables 1-3, using PSNR, MSE, and SSIM metrics. In terms of PSNR metric, the proposed method outperforms other methods with the amount of $0.776,0.375$, and $-0.537 \mathrm{~dB}$. The PSNR metric tells the bicubic method provides better performance than the prosed method, but the visual performance comparison informs the proposed method yields better result. Similarly, we used MSE and SSIM metrics for performance assessment. The proposed method outperforms other methods with the amount of $-19.392,-8.506$, and 18.756 for MSE metric, 0.010, 0.024, and -0.011 for SSIM metric.

Figure 9 shows visual performance comparison in zoomed area. As described in the previous section, the nearest neighbor method is one of simplest methods which replaces every pixel with a number of pixels of the same intensity. Therefore, the restored image shows unwanted jaggedness but all pixels are original. In the result of nearest neighbor method, staircase artifacts are clearly shown. The bilinear method and the bicubic method provide blurred image. However, the proposed method generate pleasant result image that preserving details. Both methods introduce a successive change into the result even where the original pixel has discrete changes. Therefore, both methods lessens contrast which is undesirable for linear pattern images. Although bicubic method is known as superior to the bilinear method, but the difference is negligible and the complexity is higher. 


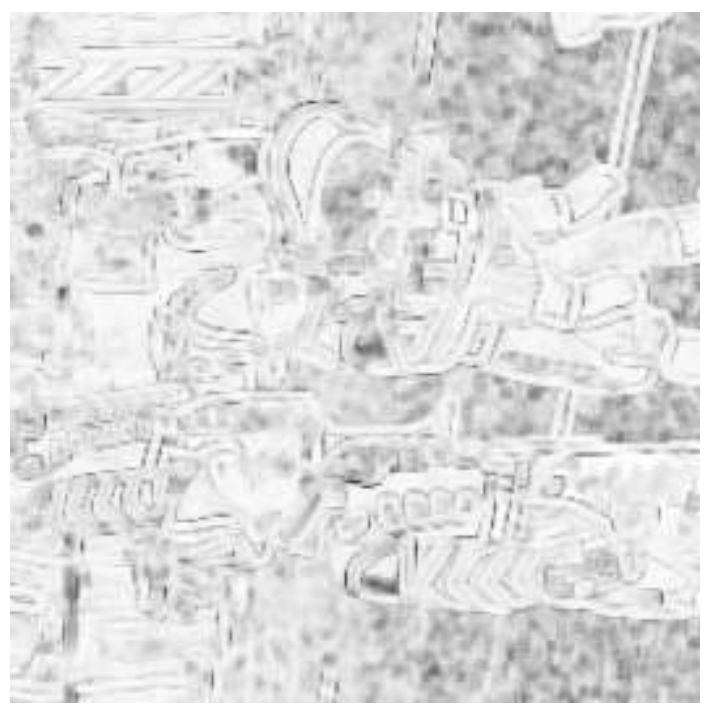

(a)

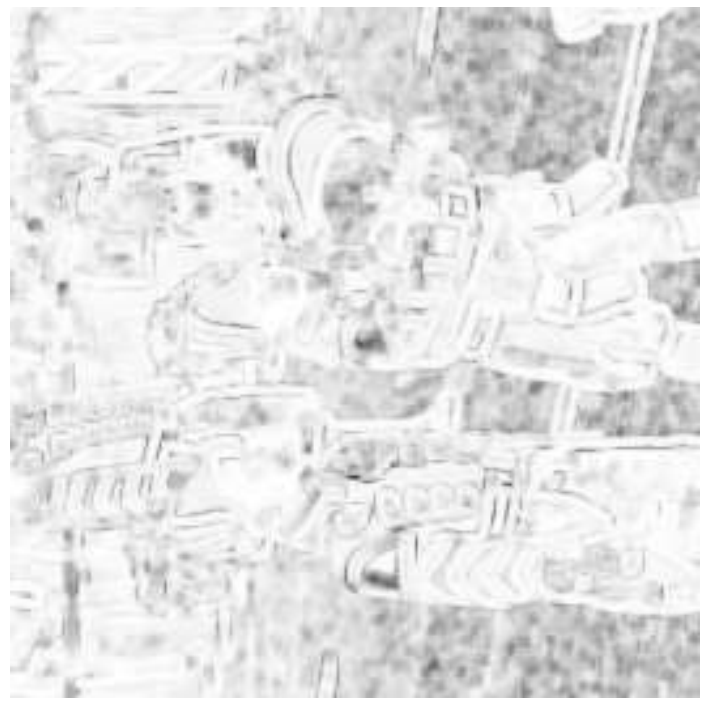

(c)

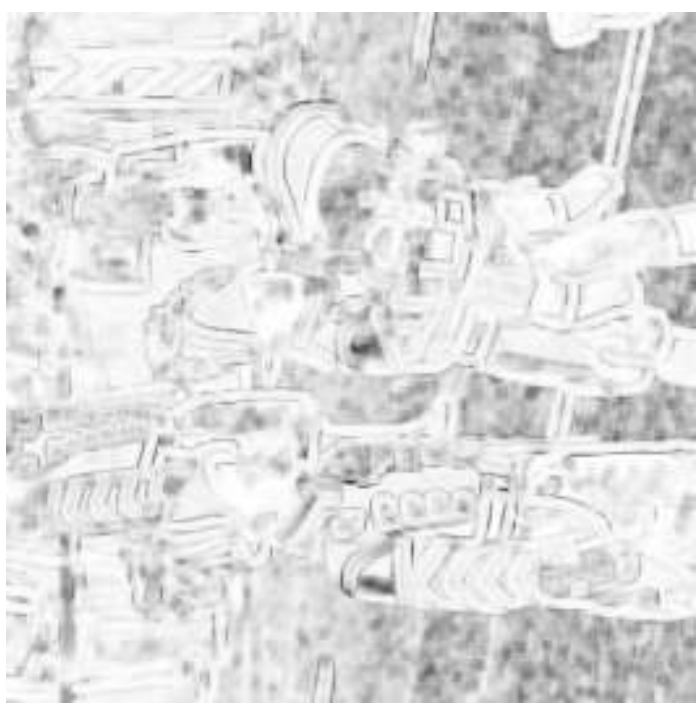

(b)

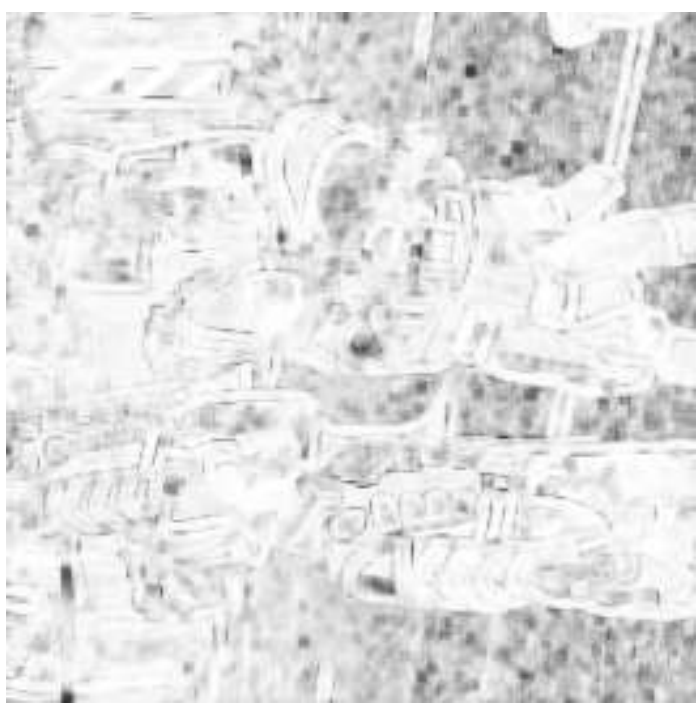

(d)

Figure 7. SSIM Map Images on \#1 LC Dataset. (a) The Nearest Neighbor Method, (b) The Bilinear Method, (c) The Bicubic Method, and (d) The Proposed Method

\section{Conclusions}

In this paper, we proposed an image resizing method. The goal of the proposed method is to rescale image size to fit the given display device. The proposed method consists of three steps, S1: least squares method based self-training, S2: image upscaling, and S3: image unsharp masking. Parameter $p$ is calculated in $\mathrm{S} 1$ and parameters $k$ and $h$ are calculated in S3. Experimental result show that the presented approach provides satisfactory results in terms of PSNR, MSE, and SSIM metrics. Moreover, subjective performance comparison tells that the proposed method is superior to the other methods. 


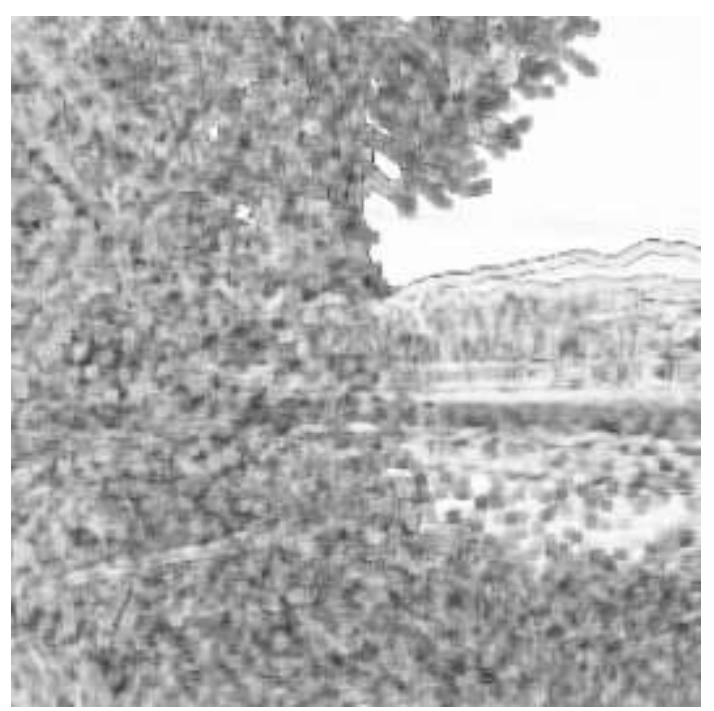

(a)

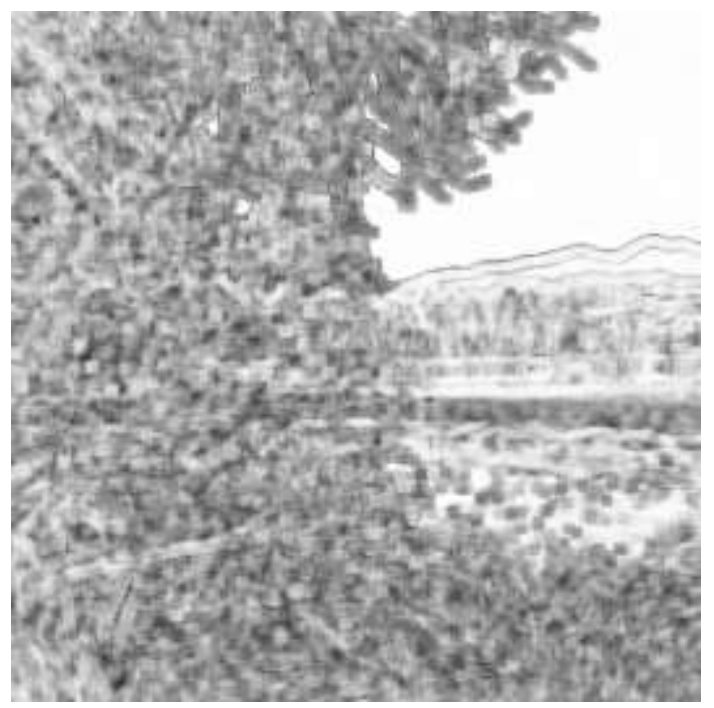

(c)

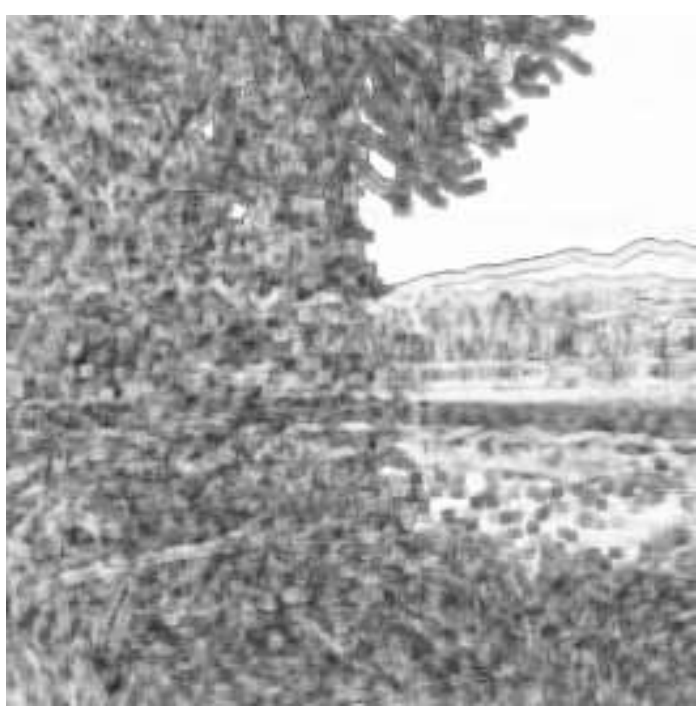

(b)

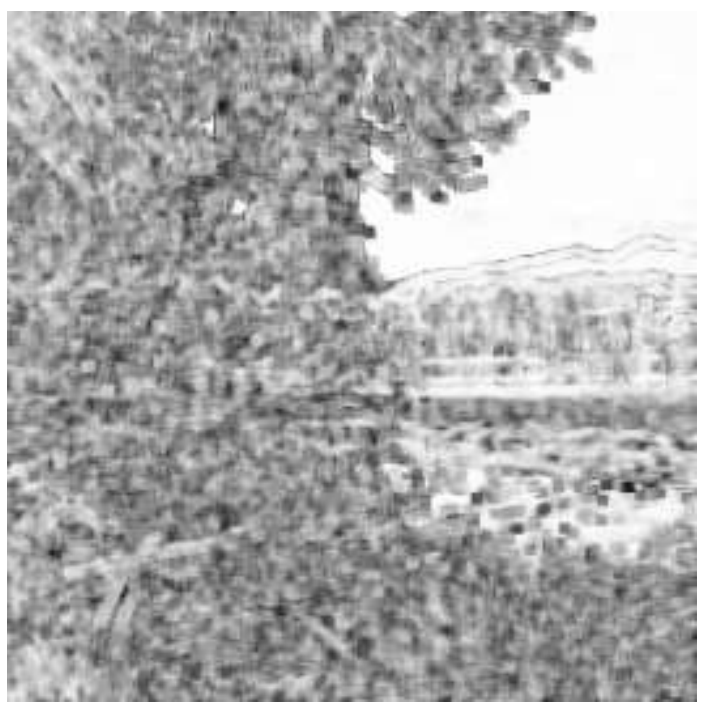

(d)

Figure 8. SSIM Map Images on \#3 LC Dataset. (a) The Nearest Neighbor Method, (b) The Bilinear Method, (c) The Bicubic Method, and (d) The Proposed Method

\section{Acknowledgements}

This work was supported by the National Research Foundation of Korea(NRF) Grant funded by the Korean Government (2015R1D1A1A01058171). This paper is a revised and expanded version of a paper entitled "Zooming Method for Higher Resolution Displays" presented at SIP2016. 
Table 1. PSNR Performance Comparison on Four Different Methods. NN: Nearest Neighbor, BI: Bilinear, BC: Bicubic, and PM: Proposed Method

\begin{tabular}{|c|c|c|c|c|}
\hline & NN & BI & BC & PM \\
\hline 1 & 25.358 & 25.887 & 26.848 & 26.367 \\
\hline 2 & 27.447 & 28.541 & 29.824 & 29.454 \\
\hline 3 & 22.191 & 22.031 & 22.547 & 21.794 \\
\hline 4 & 28.218 & 29.079 & 30.475 & 30.384 \\
\hline 5 & 27.621 & 28.069 & 29.039 & 28.559 \\
\hline 6 & 26.046 & 26.788 & 27.852 & 27.488 \\
\hline 7 & 25.167 & 25.042 & 25.644 & 24.741 \\
\hline 8 & 29.015 & 29.296 & 30.027 & 29.243 \\
\hline 9 & 23.718 & 23.855 & 24.624 & 23.941 \\
\hline 10 & 23.850 & 23.977 & 24.765 & 24.130 \\
\hline 11 & 26.864 & 27.346 & 28.365 & 27.924 \\
\hline 12 & 24.339 & 24.343 & 25.024 & 24.300 \\
\hline 13 & 30.225 & 31.180 & 32.464 & 32.263 \\
\hline 14 & 30.645 & 30.909 & 31.666 & 30.712 \\
\hline 15 & 25.553 & 25.976 & 26.862 & 26.400 \\
\hline 16 & 24.779 & 25.075 & 26.067 & 25.724 \\
\hline 17 & 26.256 & 26.702 & 27.663 & 27.329 \\
\hline 18 & 25.731 & 26.288 & 27.338 & 26.800 \\
\hline 19 & 29.623 & 29.794 & 30.440 & 29.969 \\
\hline 20 & 26.515 & 27.005 & 27.890 & 27.170 \\
\hline Avg. & 26.458 & 26.859 & 27.771 & 27.234 \\
\hline
\end{tabular}

Table 2. MSE Performance Comparison on Four Different Methods. NN: Nearest Neighbor, BI: Bilinear, BC: Bicubic, and PM: Proposed Method

\begin{tabular}{|c|c|c|c|c|}
\hline & NN & BI & BC & PM \\
\hline 1 & 189.361 & 167.655 & 134.373 & 150.114 \\
\hline 2 & 117.054 & 90.992 & 67.717 & 73.740 \\
\hline 3 & 392.597 & 407.382 & 361.738 & 430.214 \\
\hline 4 & 98.008 & 80.379 & 58.293 & 59.529 \\
\hline 5 & 112.457 & 101.439 & 81.124 & 90.612 \\
\hline 6 & 161.621 & 136.241 & 106.635 & 115.960 \\
\hline 7 & 197.890 & 203.644 & 177.277 & 218.254 \\
\hline 8 & 81.583 & 76.466 & 64.628 & 77.416 \\
\hline 9 & 276.244 & 267.655 & 224.216 & 262.390 \\
\hline 10 & 267.966 & 260.223 & 217.055 & 251.253 \\
\hline 11 & 133.870 & 119.817 & 94.754 & 104.868 \\
\hline 12 & 239.422 & 239.216 & 204.471 & 241.601 \\
\hline 13 & 61.741 & 49.554 & 36.873 & 38.615 \\
\hline 14 & 56.048 & 52.749 & 44.311 & 55.195 \\
\hline 15 & 181.051 & 164.232 & 133.932 & 148.951 \\
\hline 16 & 216.347 & 202.084 & 160.825 & 174.062 \\
\hline 17 & 153.983 & 138.951 & 111.375 & 120.278 \\
\hline 18 & 173.791 & 152.853 & 120.026 & 135.871 \\
\hline 19 & 70.921 & 68.191 & 58.756 & 65.489 \\
\hline 20 & 145.070 & 129.588 & 105.690 & 124.774 \\
\hline Avg. & 166.351 & 155.466 & 128.203 & 146.959 \\
\hline
\end{tabular}


Table 3. SSIM Performance Comparison on Four Different Methods. NN: Nearest Neighbor, BI: Bilinear, BC: Bicubic, and PM: Proposed Method

\begin{tabular}{|c|c|c|c|c|}
\hline & NN & BI & BC & PM \\
\hline 1 & 0.81328 & 0.80253 & 0.83749 & 0.82687 \\
\hline 2 & 0.86628 & 0.86787 & 0.89527 & 0.88492 \\
\hline 3 & 0.68644 & 0.63468 & 0.68952 & 0.66722 \\
\hline 4 & 0.88879 & 0.89465 & 0.92041 & 0.91575 \\
\hline 5 & 0.84512 & 0.83719 & 0.87115 & 0.86231 \\
\hline 6 & 0.89377 & 0.89662 & 0.91869 & 0.91706 \\
\hline 7 & 0.72937 & 0.68636 & 0.74292 & 0.71098 \\
\hline 8 & 0.88860 & 0.87900 & 0.89797 & 0.89110 \\
\hline 9 & 0.76651 & 0.73621 & 0.78632 & 0.76454 \\
\hline 10 & 0.83143 & 0.81376 & 0.84774 & 0.84068 \\
\hline 11 & 0.84717 & 0.83944 & 0.87055 & 0.86140 \\
\hline 12 & 0.82357 & 0.80589 & 0.83860 & 0.82775 \\
\hline 13 & 0.90698 & 0.90828 & 0.93036 & 0.92599 \\
\hline 14 & 0.87562 & 0.86451 & 0.88898 & 0.87573 \\
\hline 15 & 0.75921 & 0.72654 & 0.77699 & 0.75721 \\
\hline 16 & 0.81114 & 0.79198 & 0.84205 & 0.83848 \\
\hline 17 & 0.85708 & 0.85011 & 0.87924 & 0.87483 \\
\hline 18 & 0.86097 & 0.85650 & 0.88846 & 0.88310 \\
\hline 19 & 0.79474 & 0.77678 & 0.81416 & 0.80825 \\
\hline 20 & 0.86105 & 0.85911 & 0.88581 & 0.87634 \\
\hline Avg. & 0.83036 & 0.81640 & 0.85113 & 0.84052 \\
\hline
\end{tabular}

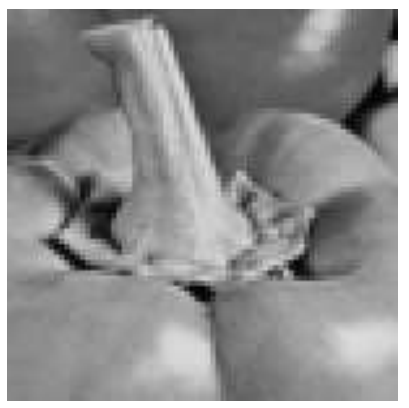

(a)

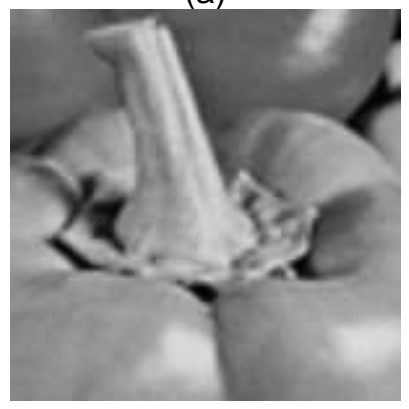

(c)

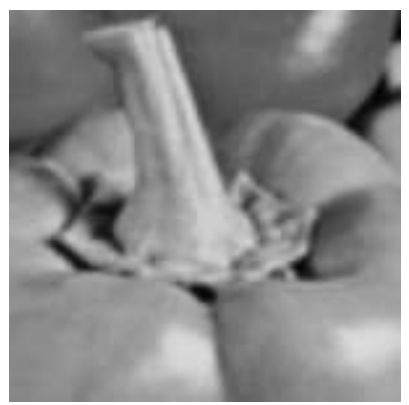

(b)

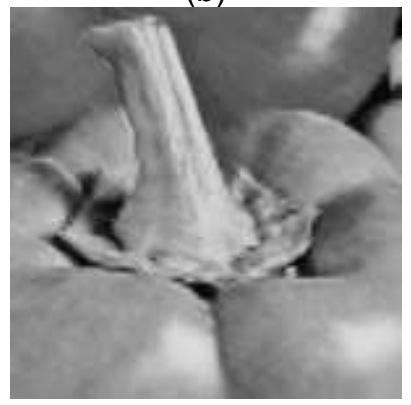

(d)

Figure 9. Visual Performance Comparison in Zoomed Area (a) Nearest Neighbor Method, (b) Bilinear Method, (c) Bicubic Method, and (d) The Proposed Method 


\section{References}

[1] R. C. Gonzalez, R. C. Gonzalez and R. E. Woods, "Digital Image Processing", Addision-Wesley, Reading MA, (1992).

[2] R. C. Tam and A. Fournier, "Image interpolation using unions of spheres," The Visual Computer, vol. 14, (1998), pp. 401-414.

[3] E. Maeland, "On the comparison of interpolation methods", IEEE Trans. Med. Image, vol. 7, no. 3, (1988), pp. 213-217.

[4] J. A Parker, R. V Kenyon and D. E Troxel, "Comparison of interpolating methods for image resampling”, IEEE Trans. Med. Image, vol. 2, no. 1, (1983), pp. 31-39.

[5] R. Adipranata, E. Cherry, G. Ballangan and R. P. Ongkodjojo, "Fast Method for Multiple Human Face Segmentation in Color Image", International Journal of Advanced Science and Technology, (2009), pp. 19-32.

[6] D. Bhattacharyya, A. Roy, P. Roy and T.-h. Kim, "Receiver Compatible Data Hiding in Color Image", International Journal of Advanced Science and Technology, (2009), pp. 15-24.

[7] B. Bhanu, J. Peng, T. Huang, and B. Draper, "Introduction to the special issue on learning in computer vision and pattern recognition”, IEEE Trans. Syst., Man, Cybern. B, Cybern., vol. 35, no. 3, (2005), pp. 391-396.

[8] J. Wang, G. Jeon, and J. Jeong, "Efficient adaptive de-Interlacing algorithm with awareness of closeness and similarity", SPIE Optical Engineering, vol. 51, no. 1, (2012), pp. 017003.

[9] X. Chen, G. Jeon, and J. Jeong, “A filter switching interpolation method for deinterlacing," SPIE Optical Engineering, vol. 51, no. 10, (2012), pp. 107402.

[10] S. J. Park, G. Jeon, and J. Jeong, "Covariance-based adaptive deinterlacing method using edge map," in Proc. IEEE IPTA2010, Paris, France, (2010), pp. 166-171.

[11] S. J. Park, G. Jeon, and J. Jeong, "Deinterlacing algorithm using edge direction from analysis of the DCT coefficient distribution", IEEE Trans. Consumer Electronics, vol. 55, no. 3, (2009), pp. 1674-1681.

[12] G. Jeon, S. J. Park, Y. Fang, M. Anisetti, V. Bellandi, E. Damiani, and J. Jeong, "Specification of efficient block matching scheme for motion estimation in video compression", SPIE Optical Engineering, vol. 48, no. 12, (2009), pp. 127005.

[13] J. Wu, Z. Xu, G. Jeon, X. Zhang, and L. Jiao, "Arithmetic coding for image compression with adaptive weight-context classification”, Signal Processing: Image Communication, vol. 28, no. 7, (2013), pp. 727-735.

[14] X. Chen, G. Jeon, and J. Jeong, "Voting-based directional interpolation method and its application to still color image demosaicking", IEEE Trans. Circuits and Systems for Video Technology, vol. 24, no. 2, (2014), pp. 255-262.

[15] X. Chen, G. Jeon, J. Jeong, and L. He, "Multidirectional weighted interpolation and refinement method for Bayer pattern CFA demosaicking", IEEE Trans. Circuits and Systems for Video Technology, vol. 25, no. 8, (2015), pp. 1271-1282.

\section{Author}

Gwanggil Jeon, received the $\mathrm{BS}$, MS, and $\mathrm{PhD}$ (summa cum laude) degrees in Department of Electronics and Computer Engineering from Hanyang University, Seoul, Korea, in 2003, 2005, and 2008, respectively. From 2008 to 2009, he was with the Department of Electronics and Computer Engineering, Hanyang University, from 2009 to 2011, he was with the School of Information Technology and Engineering (SITE), University of Ottawa, as a postdoctoral fellow, and from 2011 to 2012, he was with the Graduate School of Science \& Technology, Niigata University, as an assistant professor. $\mathrm{He}$ is currently an associate professor with the Department of Embedded Systems Engineering, Incheon National University, Incheon, Korea. His research interests fall under the umbrella of image processing, particularly image compression, motion estimation, demosaicking, and image enhancement as well as computational intelligence such as fuzzy and rough sets theories. He was the recipient of the IEEE Chester Sall Award in 2007 and the 2008 ETRI Journal Paper Award. 
International Journal of Signal Processing, Image Processing and Pattern Recognition Vol. 10, No. 6 (2017) 